\title{
Antioxidant glutathione inhibits inflammation in synovial fibroblasts via PTEN/PI3K/AKT pathway: An in vitro study
}

\author{
Wen Ting Hao'(1), Lu Huang ${ }^{1}\left(\mathbb{D}\right.$, Wei $\operatorname{Pan}^{2}$ (D), Yi Le Ren ${ }^{1}(\mathbb{D}$ \\ ${ }^{1}$ Department of Rheumatology and Immunology, Xuzhou Municipal Hospital Affiliated to Xuzhou Medical University, Xuzhou, China \\ ${ }^{2}$ Department of Pathogen Biology and Immunology, Jiangsu Key Laboratory of Immunity and Metabolism, \\ Xuzhou Medical University, Xuzhou, Jiangsu
}

\begin{abstract}
Objectives: In this study, we aimed to investigate whether glutathione (GSH) could decrease the secretion of reactive oxygen species (ROS), reduce inflammation, and modulate the phosphatase and tensin homolog deleted on chromosome 10/phosphatidylinositol 3-kinase/AKT (PTEN/PI3K/AKT) in synovial fibroblasts (SFs).

Materials and methods: A total of $30 \mathrm{DBA} / 1 \mathrm{~J}$ female mice were used in this study. The release of ROS in MH7A cells was examined using a ROS assay kit. The effects of GSH on the messenger ribonucleic acid (mRNA) expression and protein levels of inflammatory cytokines were determined via reverse transcription-quantitative polymerase chain reaction (RT-qPCR) and enzyme-linked immunosorbent assay (ELISA) in mouse SFs and MH7A cells, respectively. The PTEN/PI3K/AKT pathway was investigated via Western blotting. The effects of buthionine-sulfoximine (BSO), as an inhibitor of GSH, on these molecules were examined.

Results: The ROS were decreased after GSH treatment, and the mRNA levels of tumor necrosis factor-alpha (TNF- $\alpha$ ), interleukin (IL)-1 $\beta$, IL-6, matrix metalloproteinase (MMP)-1, MMP-3, were also significantly inhibited after GSH stimulation. However, the IL-10 levels were enhanced, and GSH increased the expression of PTEN. The GSH suppressed the activation of phosphorylated ( $p$ )-PI3K and p-AKT. The supplementation of the BSO restored the activation of PI3K/AKT pathway with a high production of ROS. The levels of TNF- $\mathrm{a}$, IL-1 $\beta$ and IL- 6 were also elevated, when the BSO was added.

Conclusion: These findings suggest that GSH can act as an inflammatory suppressor by downregulating the PTEN/PI3K/AKT pathway in MH7A cells. These data indicated a novel function of GSH for improving the inflammation of RA SFs and may help to alleviate the pathological process of RA. Keywords: Inflammation, PTEN/PI3K/AKT, reduced glutathione, rheumatoid arthritis, synovial fibroblasts.
\end{abstract}

Glutathione (GSH), a tripeptide, is an important intracellular antioxidant buffer which resist oxidative stress. It mainly exists in the forms of reduced GSH and oxidized GSH (GSSG). The metabolism of GSH is tightly controlled to maintain redox balance. However, it has been demonstrated to be constitutively lacking in patients with rheumatoid arthritis (RA), ${ }^{1,2}$ but reactive oxygen species (ROS) exhibit a higher level than normal cells. Growing numbers of studies have implicated that ROS have a close association with the accelerated inflammation of autoimmunity. ${ }^{3}$ Abundant ROS increases the expression of pro-inflammatory cytokine tumor necrosis factor-alpha (TNF- $\alpha$ ), the cytokines, in turn, aggravate over-expression of ROS, contributing to a more severe inflammatory response. In $\mathrm{RA}, \mathrm{ROS}$ are thought to directly

Received: July 23, 2021 Accepted: September 23, 2021 Published online: December 24, 2021

Correspondence: Yi Le Ren, MD. Daxue Road 269, Xuzhou 221100, Jiangsu Province, China. Tel: 18361200785 e-mail: renyile0124@163.com

\section{Citation:}

Hao WT, Huang L, Ren YL, Pan W. Antioxidant glutathione inhibits inflammation in synovial fibroblasts via PTEN/PI3K/AKT pathway: An in vitro study. Arch Rheumatol 2022;37(2):212-222. 
contribute to destructive and proliferative synovitis and, thus, play an essential role in pathophysiology of RA..$^{3,4}$ Moreover, high levels of ROS can contribute to osteoclast differentiation via RANKL signaling, inducing bone and cartilage destruction. Furthermore, ROS levels positively correlate with disease activity. 5,6

Previous studies have shown that ROS-associated transduction of signaling pathways participate in pathogenesis of RA. ${ }^{4}$ The ROS directly activate PI3K and increase downstream signaling. Concurrently, ROS inactivate tensin homolog (PTEN), which is involved in negatively regulated PIP3 synthesis and, thus, inhibits AKT activation. ${ }^{4,7}$ The PTEN is regarded as a key regulator for inhibiting the PI3K/AKT pathway. In recent years, it has been reported that the PI3K/AKT pathway may be a target for RA treatment. ${ }^{8}$

Therefore, ROS require antioxidants to remove them. Oxidation scavengers include vitamin $\mathrm{C}$ and E, coenzyme Q10 and, particularly, GSH. ${ }^{9}$ In GSH metabolic processes, different antioxidant enzymes, such as superoxide dismutase, GSH peroxidase and catalase, are found to be useful for protecting against ROS. However, as aforementioned, most of the studies have pointed out that GSH depletion is a mainstream in the pathogenesis of several inflammatory diseases. Accumulated data have shown that antioxidant supplementation may be beneficial in the treatment of autoimmune diseases. ${ }^{1,10}$

During recent years, there have been some reports to investigate how ROS-mediated cellular signaling involve in pathogenesis of RA. However, the role of antioxidant buffer GSH on ROS signals in RA synovial fibroblasts (RA SFs) still remains to be understood. In the present study, therefore, we aimed to elucidate the impact of $\mathrm{GSH}$ on the secretion of ROS, the expression of PTEN/PI3K/AKT signals and inflammatory factors in RA SFs.

\section{MATERIALS AND METHODS}

A total of $30 \mathrm{DBA} / 1 \mathrm{~J}$ female mice were used in this study (from Shanghai Laboratory Animal Center, Shanghai, China). At the time of the experiments, the mice were six to eight weeks old with a mean \pm standard error of the mean (SEM) body weight of $20 \pm 2 \mathrm{~g}$. All mice were housed in an air-conditioned room at $24^{\circ} \mathrm{C}$ with a $12 \mathrm{~h}$ dark/light cycle and permitted free access to standard laboratory food and water ad libitum. Animals were randomly divided into three groups for isolating mouse SFs under the same conditions.

Mouse SFs were separated from DBA/1J mouse knees, ankle joints and feet by cutting the synovium and bone tissue into germfree pieces. Then, these tissues were enzymatically dispersed using collagenase IV (Worthington Biochemical Corp., NJ, USA). Cells at passages 3 to 4 and 80 to $90 \%$ confluence were used for subsequent experiments. The cells were cultured at $37^{\circ} \mathrm{C}$ in a humidified atmosphere containing $5 \% \mathrm{CO}_{2}$ and with high glucose-containing DMEM (HyClone; Logan, UT, USA) supplemented with 10\% FBS (Gibco; Thermo Fisher Scientific Inc., MA, USA) and $1 \%$ penicillin-streptomycin (Gibco; Thermo Fisher Scientific Inc., MA, USA).

The MH7A cell line was purchased from Shanghai Guandao Biological Engineering Co. Ltd., and the cells were incubated in conditions similar to mouse SFs.

The MH7A cells and mouse SFs were treated with indicated concentrations of GSH (Beijing Solarbio Science \& Technology Co., Ltd, China; $100 \mu \mathrm{g} / \mathrm{mL}$ ) with or without Escherichia coli lipopolysaccharide (LPS) $(100 \mathrm{ng} / \mathrm{mL})$, the control group was treated with equal amount of phosphate buffered saline (PBS) for $24 \mathrm{~h}$ at $37^{\circ} \mathrm{C}$. The supernatants were used to detect the protein levels of cytokines via enzymelinked immunosorbent assay (ELISA). The cells were used to detect the messenger ribonucleic acid (mRNA) expression levels of cytokines via reverse transcription-quantitative polymerase chain reaction (RT-qPCR) and were used to measure the protein expression levels of PTEN, phosphorylated (p)-PI3K, and p-AKT via Western blotting.

The levels of ROS were determined using a ROS assay kit (Cat. No. S0033; Beyotime Institute of Biotechnology, Shanghai, China) according to the manufacturer's instructions. Cellular ROS production was measured using a $2^{\prime}, 7^{\prime}$-dichlorofluorescein diacetate (DCFDA) assay kit according to the manufacturer's instructions. 
The DCFDA is a cell permeable fluorogenic dye that measures hydroxyl, peroxyl, and activity of ROS within the cell. Briefly, dilute DCFH-DA with serum-free culture medium at a ratio of $1: 1000$ to a final concentration of $10 \mu \mathrm{moL} / \mathrm{L}$. Remove the cell culture medium and add $500 \mu \mathrm{L}$ diluted DCFH-DA in 24-well plate. Incubate for 20 min at $37^{\circ} \mathrm{C}$ in a humidified atmosphere $\left(5 \% \mathrm{CO}_{2}\right)$. Wash the cells three times with serum-free cell culture medium to fully remove the redundant DCFH-DA. Finally, capture the figures with laser confocal microscope.

The RNA was isolated from MH7A cells and mouse SFs using TRIzol $^{\circledR}$ reagent (Invitrogen; Thermo Fisher Scientific Inc., MA, USA), and RT was conducted using PrimeScript ${ }^{\mathrm{TM}}$ RT Master mix (Takara Bio Inc., Kusatsu, Shiga, Japan). Then, complementary deoxyribonucleic acid (cDNA) was amplified using SYBR $^{\circledR}$ Premix Ex Taq $^{\mathrm{TM}}$ (Takara Bio Inc., Kusatsu, Shiga, Japan) with gene-specific primers. The RT-qPCR analyses were performed in a LightCycler ${ }^{\circledR} 480$ II detection system (Roche Diagnostics GmbH, Roche Applied
Science, Mannheim, Germany) under the following thermal cycler conditions: initial denaturation for $5 \mathrm{~min}$ at $95^{\circ} \mathrm{C}$, followed by 45 cycles for $15 \mathrm{sec}$ at $95^{\circ} \mathrm{C}, 15 \mathrm{sec}$ at $60^{\circ} \mathrm{C}$ and $15 \mathrm{sec}$ at $72^{\circ} \mathrm{C}$, using the primers listed in Tables 1 and 2 . All experiments were performed in triplicate and the comparative cycling threshold values ( $\mathrm{Ct}$ values) were normalized to endogenous reference (GAPDH). The levels of mRNA expression were calculated using the $2^{-\Delta \Delta C q}$ method.

The levels of interleukin (IL)- 6 , TNF- $\alpha$, IL-10, and matrix metalloproteinase-1 (MMP-1) in cultured cell supernatants of MH7A cells were determined using the ELISA kits (IL-6, cat. no. EK106/2-96; MMP-1, cat. no. EK1M01-96; eBioscience; Thermo Fisher Scientific Inc., MA, USA; IL-10, Cat. No. 70-EK110/2-48; TNF- $\alpha$, Cat. No. 70-EK182-48; Hangzhou Multi Sciences (Lianke) Biotech Co. Ltd., Hangzhou, China) according to manufacturer's instructions.

For Western blotting, all proteins were isolated using the RIPA buffer (Beyotime Institute of

Table 1. Sequences for human MH7A primers (forward, F; reverse, R) used in real-time RT-qPCR

\begin{tabular}{|c|c|c|}
\hline Gene & Sequences for primers $\left(5^{\prime}-3^{\prime}\right)$ & Temperature $\left({ }^{\circ} \mathrm{C}\right)$ \\
\hline IL-6 & $\begin{array}{l}\text { F: ACATCCTCGACGGCATCTC } \\
\text { R:TCAGGACTTTTGTACTCATCTGCAC }\end{array}$ & 60 \\
\hline TNF- $\alpha$ & $\begin{array}{l}\text { F: TTGAAGAGGACCTGGGAGTAG } \\
\text { R:CGAGTGACAAGCCTGTAGCC }\end{array}$ & 60 \\
\hline IL-1 $1 \beta$ & $\begin{array}{l}\text { F: AACAGGCTGCTCTGGGATTC } \\
\text { R: TTGGTTGTAGAGGGCAAGGACCT }\end{array}$ & 60 \\
\hline MMP1 & $\begin{array}{l}\text { F:TCTTTAAAGACAGATTCTACATGCGC } \\
\text { R: GATGTCCTTGGGGTATCCGTG }\end{array}$ & 60 \\
\hline MMP3 & $\begin{array}{l}\text { F: GTTTTGGCCCATGCCTATGC } \\
\text { R: GGTCCATAGAGGGACTGAATGC }\end{array}$ & 60 \\
\hline Visfatin & $\begin{array}{l}\text { F: CGACTCCTACAAGGTTACTCACTATAAAC } \\
\text { R: CTTCTCAAGAATGTAGTTCCATCCC }\end{array}$ & 60 \\
\hline Leptin & $\begin{array}{l}\text { F: TGGTTTCATTTCTACTGTGACTGATG } \\
\text { R: TCCTAAGCAATTGCAGAAGATAAG }\end{array}$ & 60 \\
\hline Adiponectin & $\begin{array}{l}\text { F: CTTACGTTACTATCCCCAACATGC } \\
\text { R: TACACCTGGAGCCAGACTTGG }\end{array}$ & 60 \\
\hline Resistin & $\begin{array}{l}\text { F: GCAATAAGCAGCATTGGCC } \\
\text { R: CCAGGTTTATTTCCAGCTCCC }\end{array}$ & 60 \\
\hline GAPDH & $\begin{array}{l}\text { F: GGAGTCCACTGGCGTCTT } \\
\text { R: AGGCTGTTGTCATACTTCTCAT }\end{array}$ & 60 \\
\hline
\end{tabular}




\begin{tabular}{|c|c|c|}
\hline Gene & Sequences for primers(5'-3') & Temperature $\left({ }^{\circ} \mathrm{C}\right)$ \\
\hline IL-6 & $\begin{array}{l}\text { F: CCACGGCCTTCCCTAC } \\
\text { R:AAGTGCATCATCGTTGT }\end{array}$ & \\
\hline TNF- $\alpha$ & $\begin{array}{l}\text { F: CATCTTCTCAAAATTCGAGTGACAA } \\
\text { R: TGGGAGTAGACAAGGTACAACCC }\end{array}$ & \\
\hline IL-10 & $\begin{array}{l}\text { F:ACCTGGTAGAAGTGATGCCCCAGG CA } \\
\text { R: CTATGCAGTTGATGAAGATGTCAA A }\end{array}$ & \\
\hline GAPDH & $\begin{array}{l}\text { F: CAACTTTGGCATTGTGGAAGG } \\
\text { R: ACACATTGGGGGTAGGAACAC }\end{array}$ & \\
\hline
\end{tabular}

Biotechnology, Shanghai, China) and analyzed with bicinchoninic acid protein concentration assay kit (Beyotime Institute of Biotechnology, Shanghai, China). Equal amounts of protein $(\sim 60 \mu \mathrm{g})$ were subjected to SDS-PAGE $(10 \%)$ and were, then, transferred to PVDF membranes, and membranes were blocked with $5 \%$ skim milk powder and incubated in a shaker at room temperature for $4 \mathrm{~h}$ and, then, membranes were incubated with the primary antibodies (rabbit PTEN, Cat. No. 9559; p-PI3K, Cat. No. 4257; p-AKT, Cat. No. 4060; GAPDH, Cat. No. 2118; Cell Signaling Technology, Inc.; 1:1,000. $\beta$-actin, cat. no. ab008; Hangzhou Multi Sciences (Lianke) Biotech Co. Ltd., Hangzhou, China $1: 2,000$.) at $4^{\circ} \mathrm{C}$ for $12 \mathrm{~h}$. The secondary antibodies (Cat. No. GAR007 anti-rabbit IgG; Hangzhou Multi Sciences (Lianke) Biotech Co. Ltd., Hangzhou, China 1:2,000) were incubated at room temperature for $2 \mathrm{~h}$. The membranes containing antibody protein complexes were visualized with an enhanced chemiluminescence detection system on radiograph film (Bio-Rad Laboratories Inc., CA, USA). The bands were scanned and analyzed using Quantity ONE software (version 1.0; Bio-Rad Laboratories Inc., CA, USA). The expression levels of proteins in each sample were normalized to $\beta$-actin or GAPDH.

For buthionine-sulfoximine (BSO) stimulation, BSO was purchased from the Selleck Chemicals LLC, TX, USA. The MH7A cells were treated with LPS (100 ng/mL), GSH $(100 \mu \mathrm{g} / \mathrm{mL})$, LPS+GSH, LPS+BSO $(4 \mu \mathrm{M}), \mathrm{LPS}+\mathrm{BSO}+\mathrm{GSH}$ or PBS (control group) for $24 \mathrm{~h}$ at $37^{\circ} \mathrm{C}$. The collected supernatants and cells were used to the following experiments.

\section{Statistical analysis}

Statistical analysis was performed using the SPSS version 16.0 software (SPSS Inc., Chicago, IL, USA). Descriptive data were expressed in means \pm standard deviation (SD). One-way analysis of variance (ANOVA) with LSD or S-N-K post-hoc test was used to compare multiple groups. Normality and homogeneity of variances of data were established before using the oneway ANOVA analysis. A $p$ value of $<0.05$ was considered statistically significant.

\section{RESULTS}

\section{Effects of GSH on the release of ROS in MH7A}

The GSH acts as an antioxidant and decreases the release of ROS. ${ }^{11}$ In this study, the results demonstrated that the mean fluorescence intensity, representing ROS, in the LPS+GSH group was significantly lower compared to that in the LPS group (Figure 1).

\section{Effects of GSH on the mRNA levels of pro-infammatory cytokines in LPS-induced MH7A cells and mouse SFs}

The TNF- $\alpha$, IL-6, and IL-10 are implicated in the pathogenesis of RA. ${ }^{12}$ To investigate the effects of GSH and determine its appropriate dose on LPS-induced pro-inflammatory cytokine production, mouse SFs were 
PBS

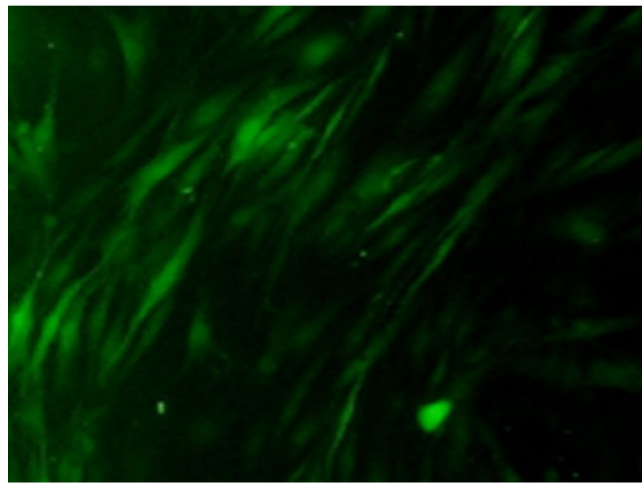

GSH
LPS

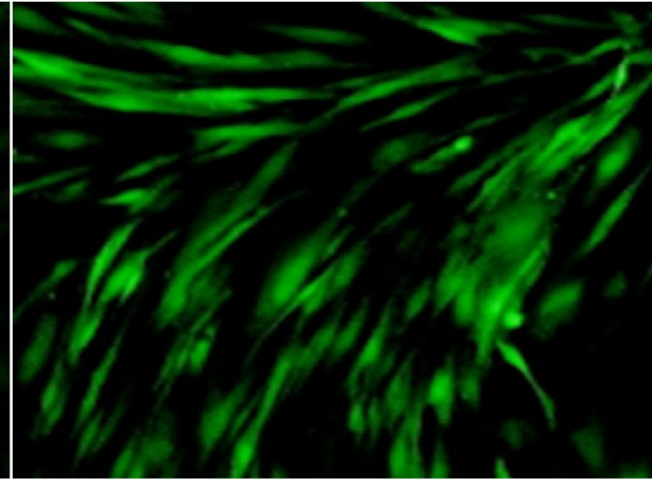

$\mathrm{LPS}+\mathrm{GSH}$
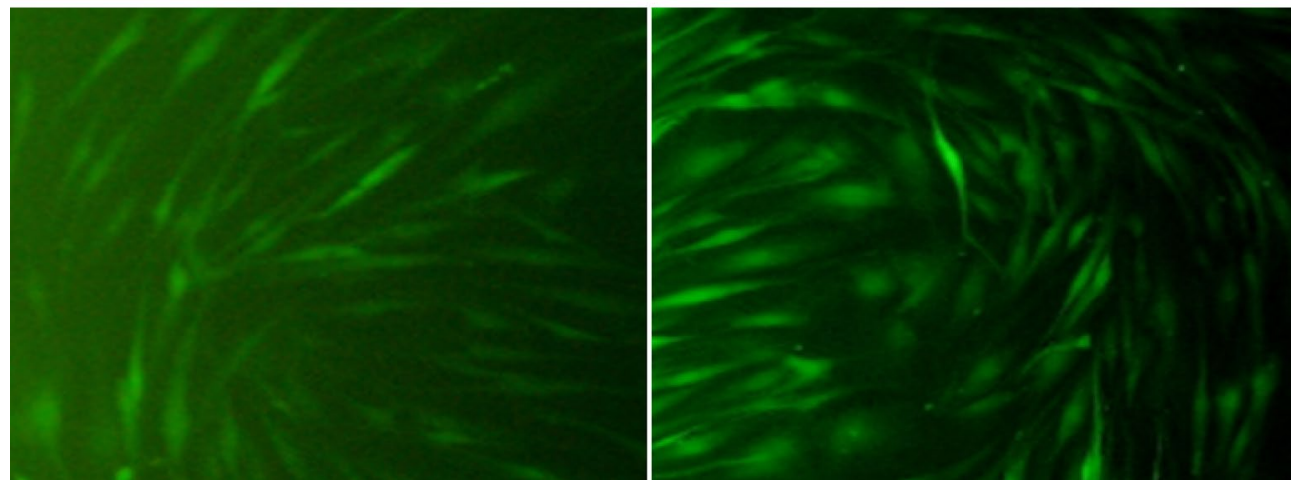

Figure 1. The effects of reduced glutathione (according to preliminary results, choose $100 \mu \mathrm{g} / \mathrm{mL}$ ) on the secretion of reactive oxygen species in human rheumatoid arthritis cell lines MH7A. The mean fluorescence intensity was detected by $\mathrm{B} \times 51$-positive fluorescence microscope $(n=3, \times 40)$.

PBS: Phosphate buffered saline; LPS: Escherichia coli lipopolysaccharide; GSH: Glutathione.

pretreated with different concentrations of GSH ranging from 50 to $150 \mu \mathrm{g} / \mathrm{mL}$ with or without LPS for $24 \mathrm{~h}$. As presented in Figure $2 \mathrm{~A}$, the results of RT-qPCR showed that GSH, only at $100 \mu \mathrm{g} / \mathrm{mL}$, significantly decreased the release of IL- 6 in LPS-stimulated mouse SFs $(p<0.05)$. Meanwhile, the mRNA level of TNF- $\alpha$ was significantly decreased, while IL-10 was enhanced $(p<0.05)$.

Figure 2B depicts further validation of the antiinflammatory effect of the selected concentration of GSH $(100 \mu \mathrm{g} / \mathrm{mL})$ in SFs. As presented, IL-6 and TNF- $\alpha$ mRNA levels were significantly decreased in the LPS+GSH group compared to the LPS group ( $p<0.05)$, whereas IL-10 was significantly elevated in the LPS+GSH group compared to the LPS group $(p<0.05)$ in mouse SFs. Furthermore, GSH $(100 \mu \mathrm{g} / \mathrm{mL})$ exhibited a significantly inhibitory effect on the mRNA levels of IL-6 and TNF- $\alpha$ in MH7A cells (Figure 2c). The IL-1 $\beta$ has been shown to stimulate matrix metalloproteinase production in human RA SFs. Moreover, mature IL-1 $\beta$ can bind to the IL-1 receptor (IL-1R) present on RA SFs, thereby resulting in the activation of the NF- $\kappa \mathrm{B}$ and MAPK pathways, indicating an important pathological relevance in modulating RA inflammatory responses. ${ }^{13}$ This study showed that IL-1 $1 \beta$ mRNA level was significantly decreased in the LPS+GSH group compared to the LPS group in MH7A cells $(p<0.05)$.

\section{Effects of GSH on the mRNA levels of MMPs in LPS-induced MH7A cells}

To measure the effects of GSH on MMPs in SFs, RT-qPCR was used to examine the expression of MMPs in MH7A. As presented in Figure 3, the expression levels of MMP-1 and MMP-3 were significantly downregulated in the LPS+GSH group compared to the LPS group $(p<0.01)$. 
(a)

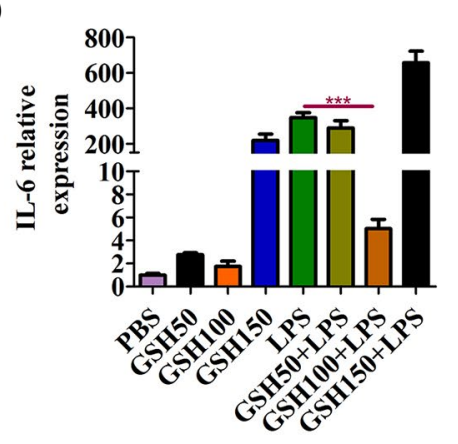

(b)

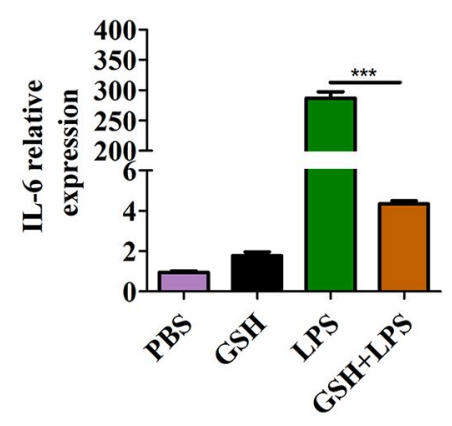

(c)

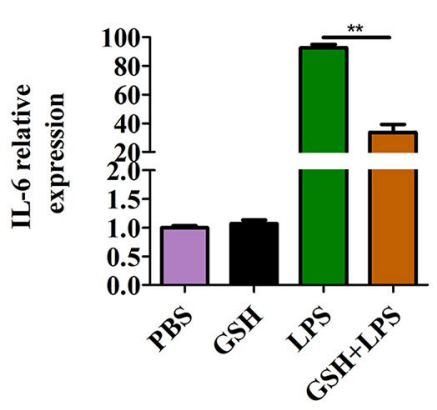

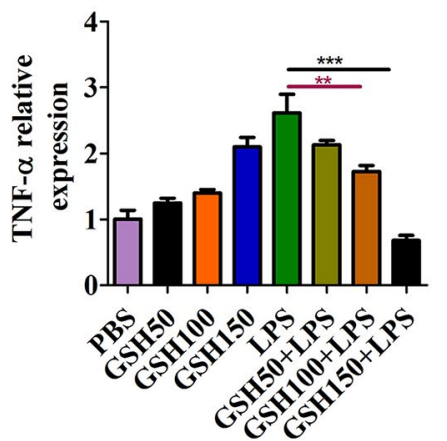
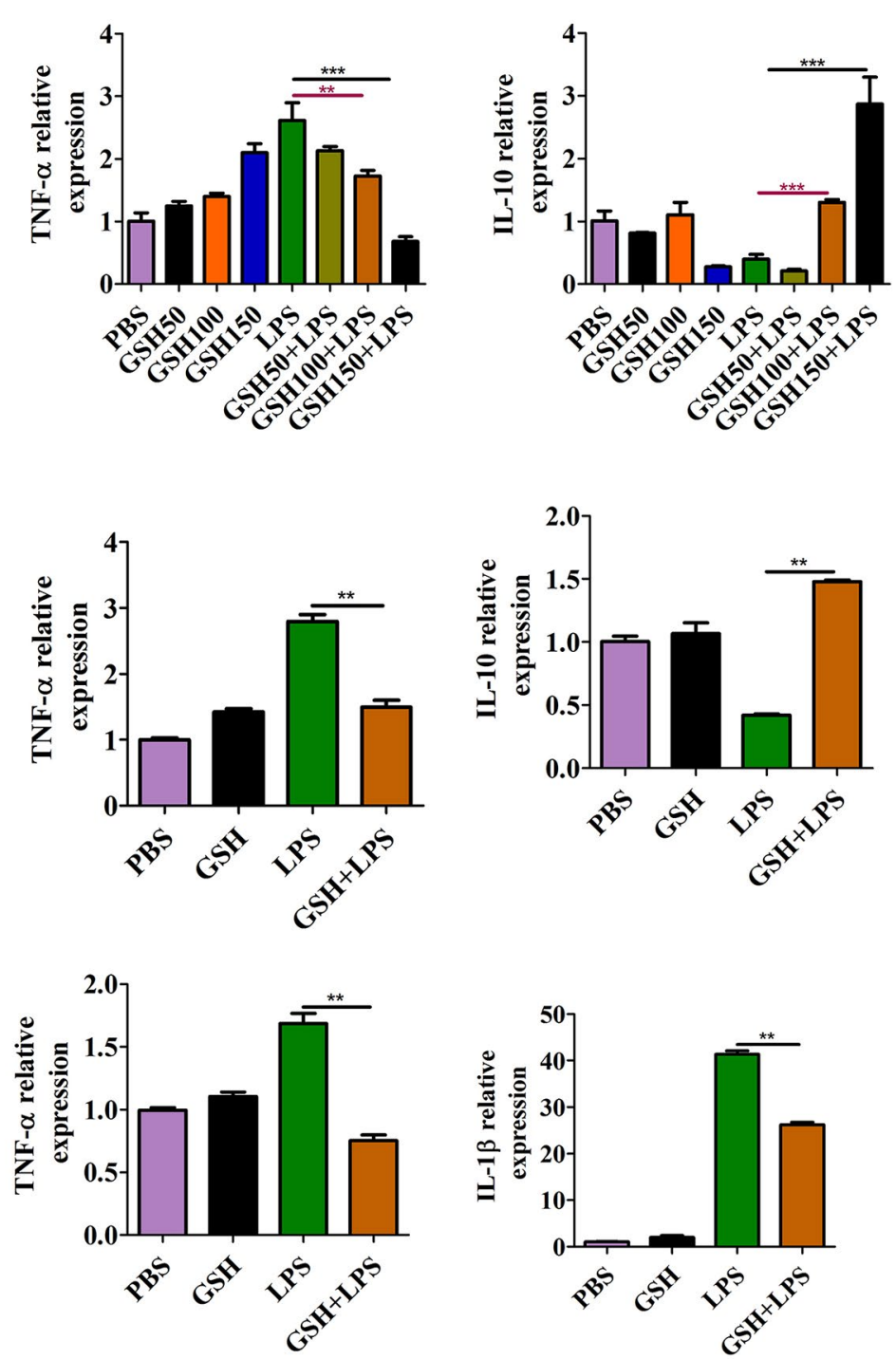

Figure 2. The effects of reduced glutathione on the release of inflammatory cytokines. (a) Reduced glutathione ranging from 50 to $150 \mu \mathrm{g} / \mathrm{mL}$ with lipopolysaccharide group compared to the lipopolysaccharide group in mouse synovial fibroblasts. (b) Reduced glutathione $100 \mu \mathrm{g} / \mathrm{mL}$ with lipopolysaccharide group compared to the lipopolysaccharide group in mouse synovial fibroblasts. (c) Reduced glutathione $100 \mu \mathrm{g} / \mathrm{mL}$ with lipopolysaccharide group compared to the lipopolysaccharide group in human rheumatoid arthritis cell lines MH7A.

Data are given in mean \pm standard deviation, unless otherwise stated. IL: Interleukin; TNF- $\alpha$ : Tumor necrosis factor-alpha; PBS: Phosphate buffered saline; LPS: Escherichia coli lipopolysaccharide; GSH: Glutathione; ${ }^{* *} \mathrm{p}<0.01 ;{ }^{* * *} \mathrm{p}<0.001$.

\section{Effects of GSH on the protein levels of cytokinesis LPS-induced MH7A cells}

At $24 \mathrm{~h}$ after $\mathrm{GSH}$ treatment, the protein levels of IL-6, TNF- $\alpha$, IL-10, and MMP-1 secreted from MH7A were evaluated via ELISA (Figure 4). The IL-6, TNF- $\alpha$, and MMP-1 levels were lower in LPS+GSH group compared to the LPS group $(p<0.05)$, However, IL-10 was significantly increased after GSH treatment. These results indicated that GSH suppressed the secretion of inflammatory cytokines and enzymes degrading collagen in MH7A.

\section{Effects of GSH on the protein levels of PTEN/PI3K/AKT pathway in MH7A cells}

To investigate the mechanism via which GSH inhibits the production of pro-inflammatory 
(a)

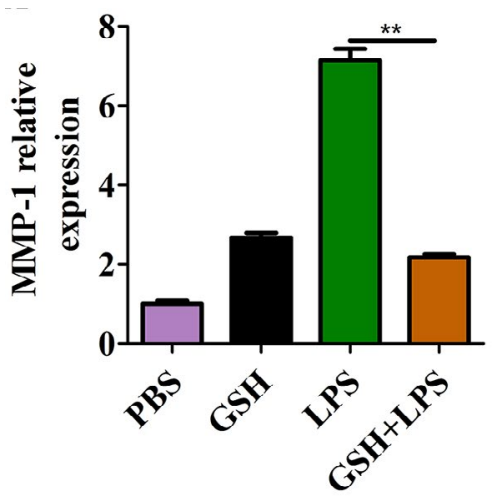

(b)

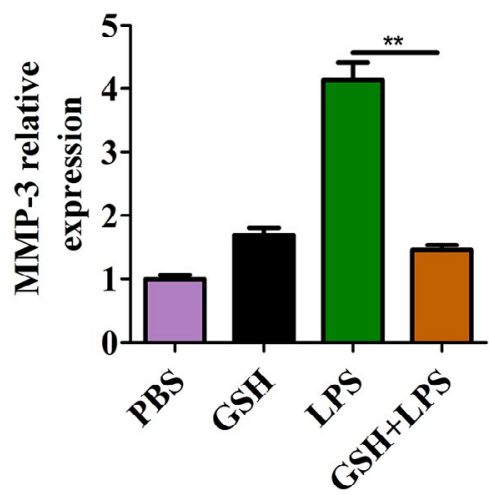

Figure 3. The effects of reduced glutathione on the release of matrix metalloproteinases. Reduced glutathione $100 \mu \mathrm{g} / \mathrm{mL}$ with lipopolysaccharide group compared to the lipopolysaccharide group in human rheumatoid arthritis cell lines MH7A.

Data are given in mean \pm standard deviation, unless otherwise stated. MMP: Matrix metalloproteinase; PBS: Phosphate buffered saline; LPS: Escherichia coli lipopolysaccharide; GSH: Glutathione; ${ }^{* *} \mathrm{p}<0.01 ;{ }^{* * *} \mathrm{p}<0.001$.
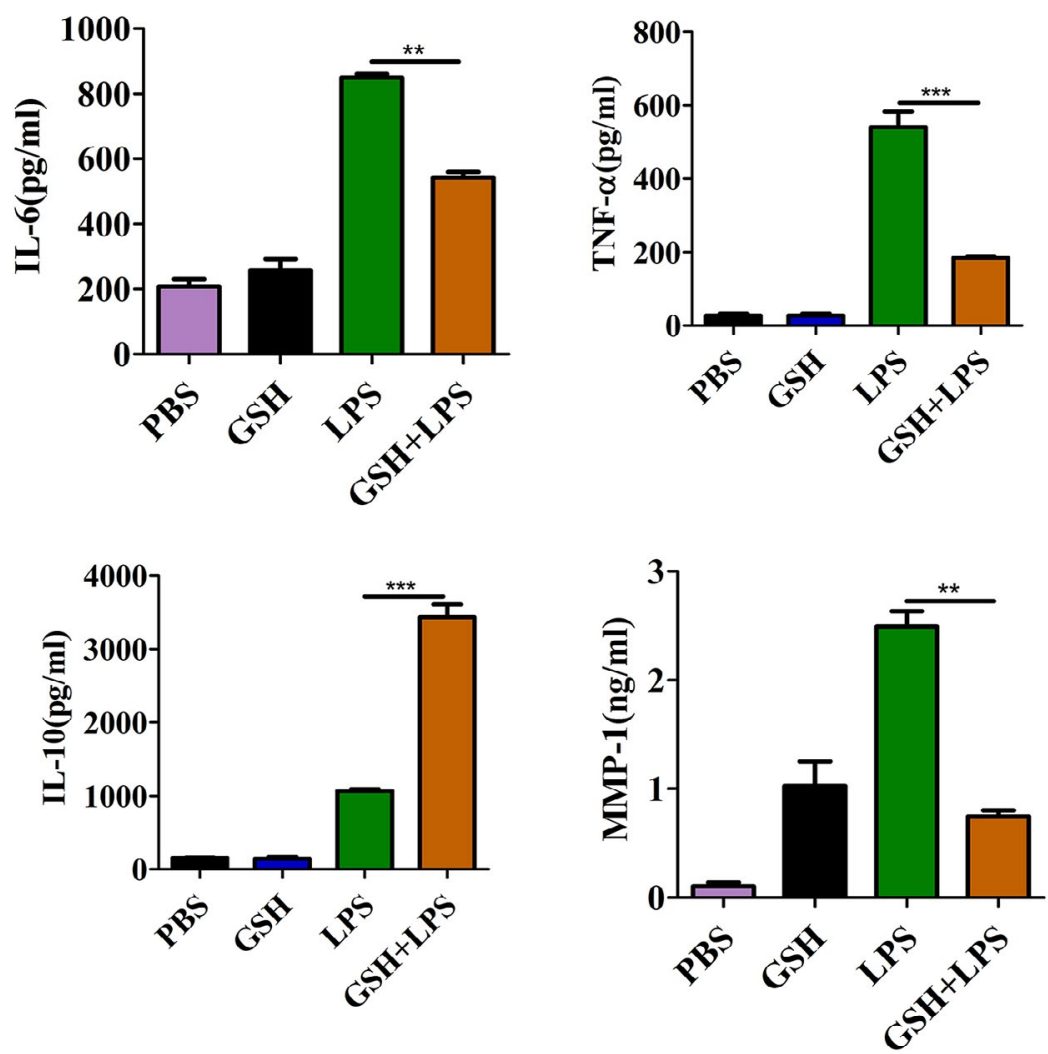

Figure 4. The effects of reduced glutathione on interleukin-6, tumor necrosis factor- $\alpha$, interleukin-10 and matrix metalloproteinases 1 in the supernatant of human rheumatoid arthritis cell lines MH7A. Reduced glutathione $100 \mu \mathrm{g} / \mathrm{mL}$ with lipopolysaccharide group compared to the lipopolysaccharide group in human rheumatoid arthritis cell lines MH7A.

Data are given in mean \pm standard deviation, unless otherwise stated. IL: Interleukin; MMP: Matrix metalloproteinase; GSH: Glutathione; ${ }^{* *} \mathrm{p}<0.01$. 


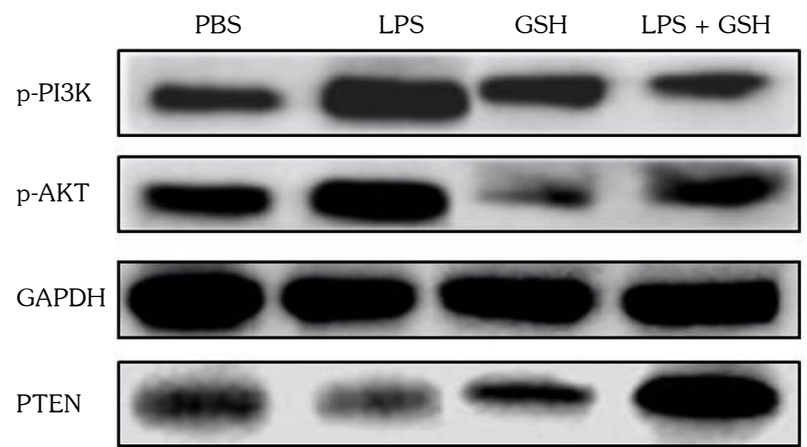

Figure 5. The effects of reduced glutathione on phosphatase and tensin homolog deleted on chromosome ten/phosphatidylinositol 3-kinase/AKT in the cell of human rheumatoid arthritis cell lines MH7A. The protein levels of phosphatase and tensin homolog deleted on chromosome ten, phosphorylated-phosphatidylinositol 3-kinase, phosphorylated-AKT were determined using Western blot $(\mathrm{n}=4)$, GSH $100 \mu \mathrm{g} / \mathrm{mL}$ with LPS group compared to LPS group in human rheumatoid arthritis cell lines MH7A.

PBS: Phosphate buffered saline; LPS: Escherichia coli lipopolysaccharide; GSH: Glutathione.

cytokines, Western blot analysis was performed to test whether GSH regulated the PTEN/PI3K/AKT signaling pathway. Regulation of PI3K/AKT pathway is usually reflected by phosphorylation of PI3K. Thus, this study detected phosphorylation of PI3K (p-PI3K) and $\mathrm{p}$-AKT protein only via Western blot. The results demonstrated that, compared to the LPS group, the expression of PTEN was enhanced, while the $\mathrm{p}-\mathrm{PI} 3 \mathrm{~K}$ and $\mathrm{p}-\mathrm{AKT}$ was significantly downregulated in the LPS+GSH group (Figure 5). These results indicated that, in MH7A cells, GSH significantly suppressed the PI3K/AKT pathway, as well as the increased PTEN expression.

\section{Effects of BSO on inflammation in MH7A cells}

After the administration $\mathrm{BSO}$, the mean fluorescence intensity was detected, and it was found that ROS levels were higher in the $\mathrm{BSO}+\mathrm{LPS}+\mathrm{GSH}$ group compared to the LPS+GSH group (Figure 6A). Furthermore, the mRNA expression levels of IL- 6 , TNF- $\alpha$, and IL-1 $\beta$ were higher in the $\mathrm{BSO}+\mathrm{LPS}+\mathrm{GSH}$ group compared to the LPS+GSH group (Figure 6b). Similarly, the mRNA expression of MMP-1 was increased after BSO treatment. In addition, Western blotting results indicated that the protein expression levels of $\mathrm{p}$-PI3K and $\mathrm{p}$-AKT were significantly upregulated, while the expression of PTEN was decreased in the LPS+GSH+BSO group compared to the LPS+GSH group (Figure 6c).

\section{DISCUSSION}

The GSH has been regarded as the principal component of the antioxidant defense system in autoimmune diseases. Previous studies have reported that the decreased levels of cellular antioxidants and elevated free radicals in the inflammatory articular cavity may lead to intensified RA. ${ }^{14}$ The ROS accumulate in the synovial fluid and peripheral blood of RA patients and can cause degenerative changes to joins, if their production and release are not balanced by sufficient antioxidants. ${ }^{15}$ The results of our study reveal that GSH in RA SFs MH7A significantly decreased the secretion of ROS, whereas the release of ROS was recovered in the presence of GSH inhibitor. An accurate redox balance, as a crucial safeguard, is necessary to sustain immunity homeostasis, which both prevent the development of autoimmunity. ${ }^{16}$ Thus, it is suggested that GSH may regulate chronic inflammation in RA by maintaining the homeostasis of ROS. However, the role of GSH in inflammation modulation of SFs is still largely unclear. In the present study, we systematically explored the role and underlying molecular mechanisms.

Several studies have shown that PTEN is a sensitive biomarker in oxidative stress..$^{17,18}$ The PTEN can suppress the activation of the PI3K/AKT pathway and exert anti-inflammatory and antioxidative stress. Downregulation of PTEN results in high levels of ROS and prominently activation of AKT. A recent study has shown that PTEN significantly reduces the arthritis and histology scores by decreasing the secretion of pro-inflammatory cytokines in collagen-induced arthritis (CIA).${ }^{19}$ Interestingly, the present study found that the protein expression level of PTEN was significantly increased in GSH-stimulated MH7A cells.

Furthermore, the abnormally activated PI3K/AKT signaling pathway is responsible for inflammation and excess proliferation of SFs in $\mathrm{RA}$. It has been previously reported that blockage of the PI3K/AKT pathway by PTEN can be a 
(a)

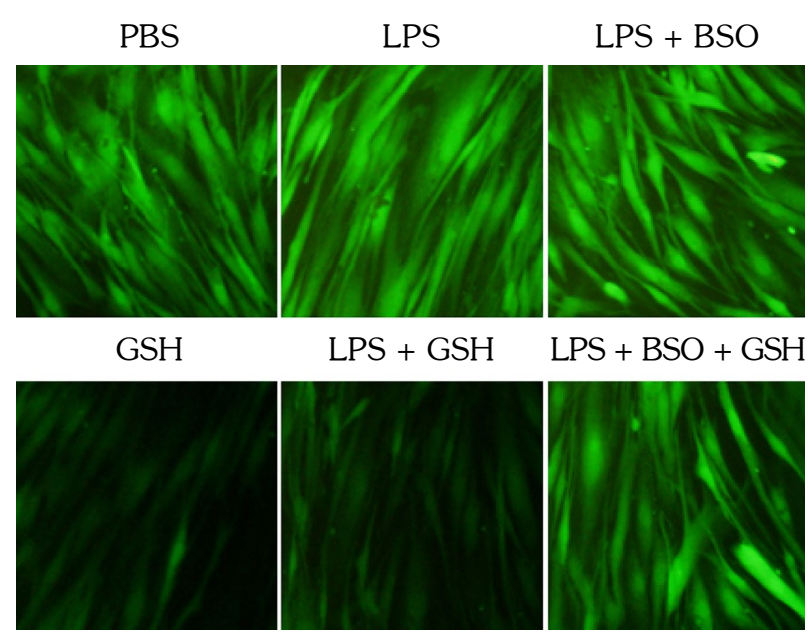

(b)
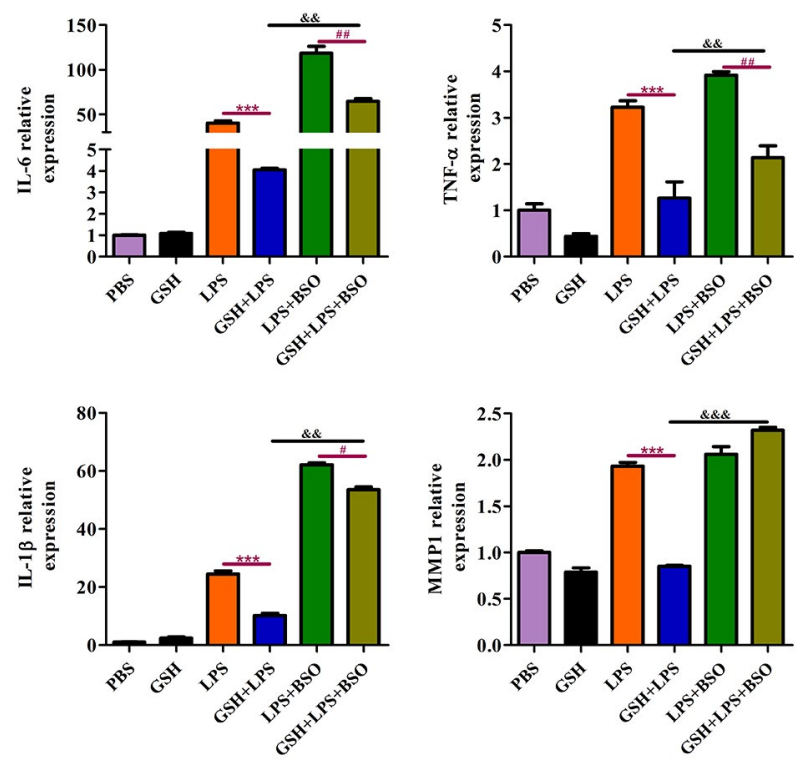

(c)
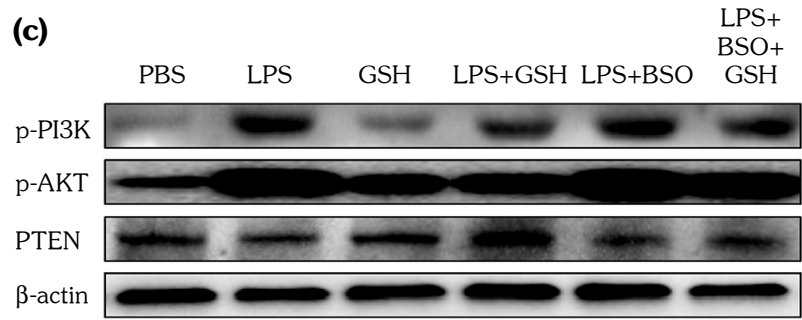

Figure 6. The effects of reduced glutathione on the inflammation in human rheumatoid arthritis cell lines MH7A while adding buthionine-sulfoximine. (a) The mean fluorescence intensity was detected by BX51-positive fluorescence microscope $(\mathrm{n}=3, \times 100)$. (b) * "reduced glutathione $100 \mu \mathrm{g} / \mathrm{mL}$ with lipopolysaccharide group compared to lipopolysaccharide group, \#reduced glutathione $100 \mu \mathrm{g} / \mathrm{mL}$ with lipopolysaccharide and buthionine-sulfoximine group compared to lipopolysaccharide and buthionine-sulfoximine group, \& reduced glutathione $100 \mu \mathrm{g} / \mathrm{mL}$ with lipopolysaccharide and buthionine-sulfoximine group compared to reduced glutathione $100 \mu \mathrm{g} / \mathrm{mL}$ with lipopolysaccharide group in human rheumatoid arthritis cell lines MH7A. ${ }^{*} \mathrm{p}<0.05,{ }^{* *} \mathrm{p}<0.01,{ }^{* * *} \mathrm{p}<0.001$. $\# \mathrm{p}<0.05, \# \mathrm{p}<0.01$. \& \& $\mathrm{p}<0.01$. (c) The protein levels of phosphatase and tensin homolog deleted on chromosome ten, phosphorylated-phosphatidylinositol 3-kinase, phosphorylated-AKT were determined using Western blot in each group from human rheumatoid arthritis cell lines MH7A. 
therapeutic target for RA. ${ }^{20}$ The present study results demonstrated the inhibitory effect of GSH on $\mathrm{p}-\mathrm{PI} 3 \mathrm{~K}$ and $\mathrm{p}$-AKT protein expression levels, as well as its promoting effect on the activation of PTEN. On the other hand, administration of the GSH inhibitor, BSO, depressed the expression of PTEN and elevated the levels of p-PI3K and $\mathrm{p}$-AKT. These findings confirm the modulation of GSH on the PTEN/PI3K/AKT pathway, which serves an important role in the mechanism of synovial inflammation. Pro-inflammatory cytokines, such as TNF- $\alpha$, IL-1 $\beta$ and IL- 6 , and MMPs secreted from SFs result in the further activation of immune cells, and the inflammatory cycle created by them leads to the pathological characters and systemic manifestations of patients with RA. ${ }^{21}$ Thus, targeting these cytokines as a therapeutic intervention has been regarded as a rational and efficient choice, and examples of such therapeutics include sarilumab, tocilizumab, infliximab, and adalimumab. ${ }^{22}$ The MMPs produced from SFs usually contribute to the destruction of cartilage seen in RA. ${ }^{23}$ The present RT-qPCR and ELISA results provided evidence that the levels of TNF- $\alpha$ and IL- 6 were significantly decreased by GSH.

However, we observed a higher level of IL-6 expression in LPS+GSH150 than that in LPS+GSH100. Meanwhile, we detected a higher IL-10 mRNA with LPS+GSH150 than with LPS+GSH100. This may result from the fact that GSH150 may pose a higher pressure to the SFs which, in turn, promotes both pro-inflammatory and anti-inflammatory cytokine expression. However, GSH100 cannot only downregulate TNF- $\alpha$ and IL- 6 expression, but also upregulate IL-10 expression, showing a strong anti-inflammatory effect. Therefore, in the present study, we used $100 \mu \mathrm{g} / \mathrm{mL}$ GSH to study the anti-inflammatory effect and underlying mechanism.

Additionally, MMPs were also decreased after GSH administration in LPS-induced MH7A cells. However, while inhibiting GSH with $\mathrm{BSO}$, the levels of these inflammatory cytokines were significantly higher than before, which indicated the inhibitory effects of GSH on inflammation in SFs. Therefore, the results suggested that GSH has a potential regulation on synovitis and bone destruction of RA by depressing cytokines and the production of ROS secreted from SFs.
Therefore, the inhibitory effect of GSH on inflammatory cytokines in MH7A cells may be associated with the enhanced expression of PTEN and the reduced expression levels of PI3K and AKT.

In recent years, there is a growing number of evidence showing that GSH system and $\mathrm{PTEN} / \mathrm{PI} 3 \mathrm{~K} / \mathrm{AKT}$ regulate each other, and the processes affect the proliferation and oxidative stress of tumor cells. ${ }^{24,25}$ However, it is still not well established on autoimmune inflammation in the synovial fibroblasts of RA. The present study showed that antioxidant GSH could decrease the levels of IL- 6 , TNF- $\alpha$, and IL- $1 \beta$ and, thus, act as an inflammatory suppressor by downregulating the ROS secretion and PTEN/PI3K/AKT pathway in synovial fibroblasts. Therefore, our data suggest that GSH may alleviate inflammation in synovial fibroblasts via reprogramming PTEN associated pathway, which provides a novel insight for explaining the anti-inflammatory mechanism for $\mathrm{GSH}$ as a drug against RA in the future.

In conclusion, to the best of our knowledge, the present in vitro study is the first to demonstrate the effect of GSH on the amelioration of the inflammatory response in MH7A and mouse SFs by targeting ROS. These findings also demonstrate the suppressive effect of GSH on the sensitive biomarker of oxidative stress, PTEN/PI3K/AKT signaling pathway, which may be regarded as a probable mechanism of GSH in inhibiting inflammation. Based on these findings, it can be considered to help the unmet needs in RA therapeutics in the future from the perspective of its antioxidant mechanism.

Ethics Committee Approval: All experiments in this study were carried out in strict compliance with the recommendations of the Guide for the Care and Use of Laboratory Animals of the Ministry of Health, China. The study protocol was approved by the Laboratory Animal Welfare and Ethics Committee (LAWEC) of Xuzhou Medical University (Xuzhou, China, SCXK (Su) 2015-0009).

Data Sharing Statement: The data that support the findings of this study are available from the corresponding author upon reasonable request.

Author Contributions: Conception and design, analysis data: W.T.H.; Analysis and interpretation of data: L.H.; Final approval of the version tobe published: W.P.; Drafting the article and revisingit: Y.L.R. 
Conflict of Interest: The authors declared no conflicts of interest with respect to the authorship and/or publication of this article.

Funding: This study was funded, in part, by the National Natural Science Foundation of China (No. 81871670), the Jiangsu Planned Projects for Postdoctoral Research Funds (No. 2019K063), and the Priority Academic Program Development of Jiangsu Higher Education Institutions. Young crops engineering reserve talent project of Xuzhou Municipal Hospital Affiliated to Xuzhou Medical University (No. QMHB2021004).

\section{REFERENCES}

1. Perricone C, De Carolis C, Perricone R. Glutathione: A key player in autoimmunity. Autoimmun Rev 2009;8:697-701.

2. Kadry MO. Liposomal glutathione as a promising candidate for immunological rheumatoid arthritis therapy. Heliyon 2019;5:e02162.

3. Hoffmann MH, Griffiths HR. The dual role of Reactive Oxygen Species in autoimmune and inflammatory diseases: Evidence from preclinical models. Free Radic Biol Med 2018;125:62-71.

4. Phull AR, Nasir B, Haq IU, Kim SJ. Oxidative stress, consequences and ROS mediated cellular signaling in rheumatoid arthritis. Chem Biol Interact 2018;281:121-36.

5. Lee NK, Choi YG, Baik JY, Han SY, Jeong DW, Bae YS, et al. A crucial role for reactive oxygen species in RANKL-induced osteoclast differentiation. Blood 2005;106:852-9.

6. Pucino V, Certo M, Varricchi G, Marone G, Ursini F, Rossi FW, et al. Metabolic checkpoints in rheumatoid arthritis. Front Physiol 2020;11:347.

7. Leslie NR, Downes CP. PTEN: The down side of PI 3-kinase signalling. Cell Signal 2002;14:285-95.

8. Yan X, Liu Y, Kong X, Ji J, Zhu H, Zhang Z, et al. MicroRNA-21-5p are involved in apoptosis and invasion of fibroblast-like synoviocytes through PTEN/ PI3K/AKT signal. Cytotechnology 2019;71:317-28.

9. Farzaei MH, Zobeiri M, Parvizi F, El-Senduny FF, Marmouzi I, Coy-Barrera E, et al. Curcumin in liver diseases: A systematic review of the cellular mechanisms of oxidative stress and clinical perspective. Nutrients 2018;10:855.

10. Gambhir JK, Lali P, Jain AK. Correlation between blood antioxidant levels and lipid peroxidation in rheumatoid arthritis. Clin Biochem 1997;30:351-5.

11. Diaz-Vivancos P, de Simone A, Kiddle G, Foyer $\mathrm{CH}$. Glutathione--linking cell proliferation to oxidative stress. Free Radic Biol Med 2015;89:1154-64.

12. Abdelmawgoud H, Saleh A. Anti-inflammatory and antioxidant effects of mesenchymal and hematopoietic stem cells in a rheumatoid arthritis rat model. Adv Clin Exp Med 2018;27:873-80.
13. Singh AK, Fechtner S, Chourasia M, Sicalo J, Ahmed S. Critical role of IL- $1 \alpha$ in IL-1 $\beta$-induced inflammatory responses: Cooperation with NF- $\mathrm{kBp} 65$ in transcriptional regulation. FASEB $\mathrm{J}$ 2019;33:2526-36.

14. Ishibashi T. Molecular hydrogen: New antioxidant and anti-inflammatory therapy for rheumatoid arthritis and related diseases. Curr Pharm Des 2013;19:6375-81.

15. Mateen S, Moin S, Khan AQ, Zafar A, Fatima N. Increased reactive oxygen species formation and oxidative stress in rheumatoid arthritis. PLoS One 2016;11:e0152925.

16. Marí M, Morales A, Colell A, García-Ruiz C, Kaplowitz N, Fernández-Checa JC. Mitochondrial glutathione: Features, regulation and role in disease. Biochim Biophys Acta 2013;1830:3317-28.

17. Zhou S, Sun Y, Zhao K, Gao Y, Cui J, Qi L, Huang L. miR-21/PTEN pathway mediates the cardioprotection of geniposide against oxidized low-density lipoprotein-induced endothelial injury via suppressing oxidative stress and inflammatory response. Int J Mol Med 2020;45:1305-16.

18. Ray PD, Huang BW, Tsuji Y. Reactive oxygen species (ROS) homeostasis and redox regulation in cellular signaling. Cell Signal 2012;24:981-90.

19. Lu H, Chen W, Liu W, Si Y, Zhao T, Lai X, et al. Molecular hydrogen regulates PTEN-AKTmTOR signaling via ROS to alleviate peritoneal dialysis-related peritoneal fibrosis. FASEB J 2020;34:4134-46.

20. Shen P, Huang Y, Ba X, Lin W, Qin K, Wang H, et al. Si Miao San attenuates inflammation and oxidative stress in rats with CIA via the modulation of the Nrf2/ARE/PTEN pathway. Evid Based Complement Alternat Med 2021;2021:2843623.

21. Cavalli G, Dinarello CA. Treating rheumatological diseases and co-morbidities with interleukin-1 blocking therapies. Rheumatology (Oxford) 2015;54:2134-44.

22. Senolt L. Emerging therapies in rheumatoid arthritis: Focus on monoclonal antibodies. F1000Res 2019;8:F1000.

23. Haque M, Singh AK, Ouseph MM, Ahmed S. Regulation of Synovial Inflammation and Tissue Destruction by Guanylate Binding Protein 5 in Synovial Fibroblasts From Patients With Rheumatoid Arthritis and Rats With Adjuvant-Induced Arthritis. Arthritis Rheumatol 2021;73:943-954.

24. Akca H, Demiray A, Aslan M, Acikbas I, Tokgun O. Tumour suppressor PTEN enhanced enzyme activity of GPx, SOD and catalase by suppression of PI3K/ AKT pathway in non-small cell lung cancer cell lines. J Enzyme Inhib Med Chem 2013;28:539-44.

25. Chen $B$, Shen $Z$, Wu D, Xie X, Xu X, Lv $\mathrm{L}$, et al. Glutathione peroxidase 1 promotes NSCLC resistance to cisplatin via ROS-induced activation of PI3K/AKT pathway. Biomed Res Int 2019;2019:7640547. 